\title{
Vaidic Care Hand Sanitizer
}

\section{Shubhada Amit Kulkarni1*, Jayshree Sharma ${ }^{3}$, Anjali Bobade ${ }^{3}$ and (Dr. Ganesh Tapdiya ${ }^{2}$ )}

${ }^{1}$ Assistant Professor, Shreeyash Institute of Pharmaceutical Education and Research, Aurangabad Maharashtra, India

${ }^{2}$ Principal Shreeyash institute of pharmaceutical education and research Aurangabad, India

${ }^{3}$ Shreeyash institute of pharmaceutical education and research Aurangabad, India

*Corresponding Author: Shubhada Amit Kulkarni, Assistant Professor, Shreeyash Institute of Pharmaceutical Education and

Research, Aurangabad Maharashtra, India.

Received: January 31, 2022; Published: February 05, 2022

DOI: 10.55162/MCPS.22.030

\begin{abstract}
Our hand carries variety of microorganisms, it's essential not to neglect them. Hands are major source of infection. Hand hygiene is a vital principle and exercise in the prevention, control and reduction of healthcare acquired infections. Right hand washing and drying methods stop the chain of transmission of deadly pathogens form hand to other parts of body. Hand sanitization is preeminent aid in preventing nosocomial infections caused by different opportunistic pathogens and to get this, the use of hand sanitizer become must in recent circumstances. The purpose of the recent study was to prepare the Ayurvedic Sanitizer- "VaidicCare". This hand sanitizer is prepared by using "Gomutra" that is cow urine and this sanitizer incorporating the leaves of Ocimum sanctum, Azadirachta indica, leaves of papaya, alovera gel, turati, flower of ajwayan, flower of pudina and Bhimsen kappor, the well-known herbal combination with multidimensional activities and to evaluate their respective antimicrobial efficiency and safety of hands.The formulation was evaluated against specified micro-organisms.

(E. coli, staphylococcus, lactobacillus, Trichoderma, aspergillus) by cup plate method. The significance was found to be more in comparison to the standard references.

Vaidiccare had its unique fragrance. Research mainly focuses on antibacterial activity after combination herbal plants cow urine and amrut dhara.Maintain hand hygiene is play key role in food production, pharmaceutical industries as well as in covid pandemic. By using vaidic care hand sanitizer we can maintain hand hygiene which is an important step to stop various infections which is caused due to unhygienic hand. Vaidic care overcomes all the side effect caused due to the chemical sanitizer.
\end{abstract} Keywords: Hand hygiene Antimicrobial Activity; Nosocomial Infection; Ayurvedic Sanitizer; Amrut Dhara; Cow Urine

\section{Introduction}

As we know skin is the most exposed part of the body to the environmental pollution sunlight and also pathogens, especially hand skin. Therefore, hand hygiene is most important major to avoid the contamination and to avoid transmission of harmful germs and to prevent infection.

Therefore, the spread of microorganism and germs can be prevented by the frequent use of hand washes and the hand sanitizer at all time washing hand is not possible so using hand sanitizer become important aspect. in the all formulation of sanitizer available in market they use number of chemicals and alcohol are mainly use which shows very good antimicrobial activity but they show plenty of side effect like it is carcinogenic it causes the hand irritation redness and it cannot be use for small children and we overcome with all this side effect cause by the alcohol and we successfully replace the alcohol in the chemical sanitizer with cow urine [1].

Replacement of alcohol with some other herbal ingredient itself it is big task and we have done the same we come up with the pure 
herbal sanitizer which is purely herbal, free from all the chemicals and alcohol though it is free from alcohol it shows a very good antimicrobial activity as chemical hand sanitizer shows. Antimicrobial activity tested against gram +ve and gram-ve bacteria some fungi $[2]$.

Vaidic care hand sanitizer contains cow urine and various herbal plant extraction example Azadirachta indica, Ocimum tenuiflorum, aloe vera, leaves of carica papaya and petals of various flowers like rose, plumerica flowers and the most important ingredient is amrut dhara contain Bhimsen Kapoor, mentha [pudina flowers], ajwain flowers vaidic care is liquid type of hand sanitizer [3-4].

Several studies suggested that sanitizer with at least70\%alcohol were suggested to kill 99.9\%of germs on hands but it is very sensitive for small babies and older patients too as in vaidic care hand sanitizer it is formulated using herbal plants and cow urine which show no side effect and which not sensitive to skin and can be use by children and older patients. It is purely herbal sanitizer [5].

\section{Procedure}

\section{Materials and Methods}

Drug

1. Leaves of Osmium Sanctum.

2. Leaves of AzadirachtaIndica.

3. Papaya Leaves.

4. Aloe Vera.

5. Patelof Flowers [ rose, plumeria].

6. Amrut dhara [ Bhimsen Kapoor, ajwain flowers, pudina flowers Turati.

Plant leaves collected from the botanical garden of shreeyash institute of pharmaceutical education and research Aurangabad.

\section{Bacterial Strains}

1. Escherichia coli [gram-ve]

2. Staphylococcus aureus [gram + ve]

3. Lactobacillus [gram +ve]

\section{Fungi Strains}

1. Aspergillus Niger

2. Trichoderma

\section{Extraction}

Extraction of phytoconstituent done directly by taking the fine paste of leaves of Ocimum tenuiflorum and Azadirachta indica and papaya leaves,aloe pulp and Patel of flowers was taken as it is [6].

\section{Formulation}

Boiled cow urine for 10 min the addition of paste of various leaves of Ocimum sanctum, Azadirachta indica,carica papaya and aloe pulp and petals of rose, plumeria directly add into mixture, allow the mixture boiled untill it become half. after that allow the mixture for cooling same time add turati into that, before starting the formulation prepare amrut dhara which contain mixture of Bhimsen Kapoor, ajwain flowers and pudina flowers [mentha]. Whole three ingredient placed in airtight container untill it liquify .then filter the mixture using the cotton cloth and last adding amrut dhara in that mixture and shake well.sanitizer is ready for evaluation. Also, we perform comparative study of herbal,chemical and vaidic care sanitizer and among that vaidic care shows most prominent activity and shows no bacterial growth [7-8]. 


\begin{tabular}{|c|l|l|}
\hline S No. & Ingredient for $\mathbf{1 0 0 0} \mathbf{~ m l}$ & Quantity taken \\
\hline 1 & Ocimum sanctum & $20 \mathrm{gm}$ \\
\hline 2 & Azadirachta indica & $20 \mathrm{gm}$ \\
\hline 3 & Carica papaya & $10 \mathrm{gm}$ \\
\hline 4 & Aloe pulp & $25 \mathrm{gm}$ \\
\hline 5 & Patel of rose and plumeria & $30 \mathrm{gm}$ \\
\hline 6 & Amrut dhara [Bhimsen Kapoor, mentha,ajwain] & $30 \mathrm{gm}[\mathrm{each} 10 \mathrm{gm}]$ \\
\hline
\end{tabular}
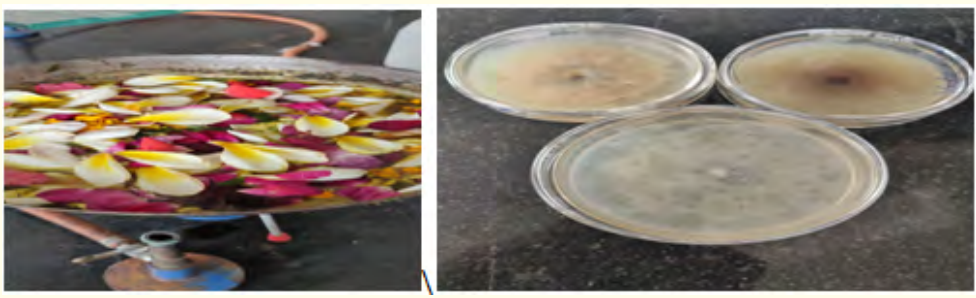

[comparative study of chemical ,herbal and vaidic care]

\section{In-Vitro Antimicrobial Activity By Agar Platediffusin [Pour Plate] Method}

In present study the antimicrobial activity of trial sanitizer was carried out by the agar plate diffusion method .the dilution of sanitizer are prepared and up to 0.10 are only affective for the antimicrobial activity. Prepare the agar petri dish and add various bacterial and fungal culture suspensions in that agar plate using pour plate method allow the agar medium for solidification. Then using cork borer perform holes on agar plate in that hole adding two to three drops of sanitizer allow it for diffusion for 24 hours after that we check that antibacterial and anti-fungal activity of sanitizer by calculating the length of zone of inhibition [9-12].

\section{Results}

Total three bacterial and two fungal species are taken in the present study to evaluate antimicrobial activity of the sanitizer. Different concentration of the sanitizer was incubated, and the dilutions are not that much affected they show antimicrobial activity only up to 0.10 concentration, and the antimicrobial activity is carried out by counting the zone of inhibition among three bacterial species [staphylococcus aureus, E. coli, lactobacilli] and fungal species [Trichoderma, aspergillusNiger] found to be greater in comparison to reference standard 70\% OF alcohol sanitizer. This indicates that the hand sanitizer has antibacterial activity particularly against all this bacterial and fungal strains.

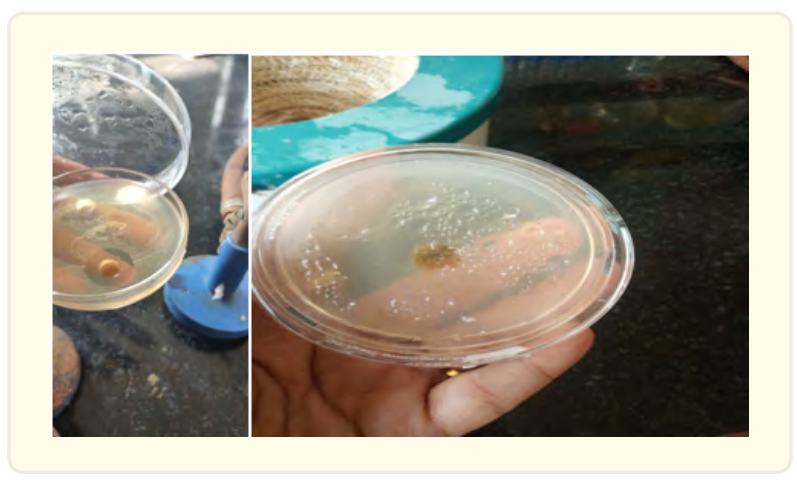




\section{Discussion}

The prepared herbal alcohol free formulation of hand sanitizer showed significant result starting from 0.10 dilution against three bacterial species and two fungal species. The significance was found to be more in comparison to standard reference .the composition [Ocimum Sanctum and AzadirachtaIndica] has been attributed with properties like free radical scavenging, anthelmintic antimicrobial anti-inflammatory and analgesic etc. more concentration may be needed to get a broad spectrum activity of the test drug.

The cow urine with all of the ingredient have excellent, rapid germicidal activity against vegetative bacteria, fungi and antimicrobial activity is based on protein denaturation of microorganism. Vaidic care is sanitizer are highly effective against mycobacteria and multidrug resistant pathogens vaidic care have an plenty of advantages like it is free from alcohol so it is not carcinogenic, nonirritant, can be easily use by the children and older patients rubbing the sanitizer on the both hand until it evaporates, usually requires 10 to 15 seconds. Whereas vigorous friction, rinsing with water, and drying with towel is not needed like hand disinfectant and soaps.

\section{Conclusion}

Hands are the most common mode of transmission of pathogens to patients and proper hand hygiene can prevent health care -associated infections and the spread of the antimicrobial resistant. Scientific evidence and ease of use support of herbal [chemical and alcohol free] hand sanitizer during patient's care. It may be concluded that herbal hand sanitizer has a significant antimicrobial effect on the specified organism. Thus, there is an immense potential in establishing the use of antimicrobial herbal product as measure to overcome all the side effect caused by the alcohol based and sanitizer.

\section{References}

1. Firdous Shaikh., et al. "Formulation and evaluation of herbal hand sanitizer using Psidium guajava leaves extract". (2020).

2. Dr kalaivani., et al. "A study on evaluation and effectiveness of herbal hand sanitizer and its anti-bacterial activity". International journal of trend in scientific research and development (IJTSRD) (2018).

3. Mithun A Thombare., et al. "Formulation and evaluation of novel herbal hand sanitizer". INDO American journal of pharmaceutical research.

4. Sajjan Shetty Mallikarjun., et al. Antimicrobial efficacy of Tulsi leaf (Ocimum Sanctum) extract on periodontal pathogens: A vitro study". Journal of Indian Society of periodontology 20.2 (2016): 145-150.

5. Mohammad A Alzohairy. "Therapeutics Role of Azadirachta indica (Neem) Their Active Constituents in Diseases prevention and Treatment". Evid Based Complement Alternat Med (2016).

6. Rubina Lawrence., et al. "Isolation, purification and evaluation of antibacterial agents from aloe vera". Brazilian journal of Microbiology -Brazilian society of microbiology 40.4 (2009): 906-15.

7. Bri B Tewari and Gomathinayagam. Subramanian and Rekha gomathinayagm, antimicrobial properties of carica papaya (papaya) different leaf extract against E. coli, S. aureus and C. albicans, American journal of pharmacology and pharmacotherapeutics.

8. Yasser shahbazi. "Chemical composition and in vitro Antibacterial activity of Mentha spicata Essential Oil against common Food -borne pathogenic bacteria, Hindawi.

9. Bhatt, Mahesh Kuma M and Periyar Selvam's. "Antimicrobial effect of ajwain seed ethanolic extract against food borne pathogenic bacteria". International food and research journal $25.3: 908-912$

10. Rashmi Yadav., et al. Health benefits of Indian aromatic plant ajwain.

11. N S Wani., et al. "Formulation and evaluation of herbal sanitizer".

12. Rutuja Sunil Patankar and Dr Naynachandak. "Formulation of herbal sanitizer and determining their antimicrobial activities against skin pathogens". International journal of innovative science and research technology.

\section{Volume 2 Issue 2 February 2022}

\section{(C) All rights are reserved by Shubhada Amit Kulkarni., et al.}

